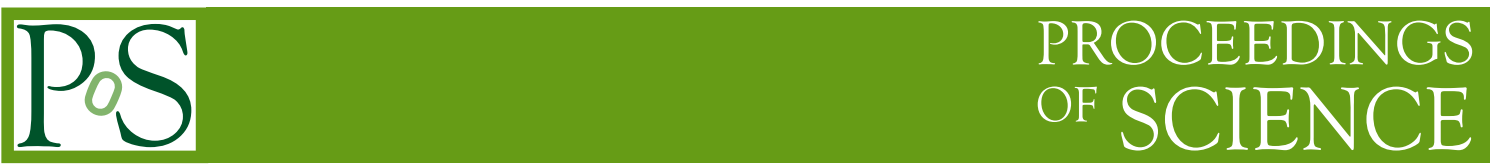

\title{
Cosmic-ray escape from supernova remnants
}

\author{
Robert Brose*, M. Pohl \\ Institute of Physics and Astronomy, University of Potsdam, Karl-Liebknecht-Str. 24/25, 14476 \\ Potsdam, Germany \\ DESY, Platanenallee 6, 15738 Zeuthen, Germany \\ E-mail: robert.brose@desy.de
}

\begin{abstract}
Supernova remnants are known to accelerate cosmic rays for their non-thermal emission of radio waves, X-rays, and gamma-rays. Although there are many models for the acceleration of cosmic rays in supernova remnants, the escape of cosmic rays from this sources is yet understudied.

We use our time-dependent acceleration code to study the acceleration of cosmic rays and their escape from supernova remnants.

We carry out spherically symmetric 1-D simulations in which we simultaneously solve the transport equations for cosmic rays, magnetic turbulence, and the hydrodynamical flow of the thermal plasma. The transport equations for cosmic-rays and magnetic turbulence are coupled via the cosmic-ray gradient and the spatial diffusion coefficient of the cosmic rays, while the cosmic-ray feedback onto the shock structure is ignored. Our simulations span 100000 years, thus covering the free-expansion, the Sedov-Taylor and the radiative phase of the remnant evolution. During this time we keep all cosmic rays in the simulation domain.
\end{abstract}

At later stages of the evolution cosmic-rays in a wide energy-band are able to escape the remnant. We compare the results with common analytic estimates for the escape-spectra.

35th International Cosmic Ray Conference - ICRC2017

10-20 July, 2017

Bexco, Busan, Korea

${ }^{*}$ Speaker. 


\section{Introduction}

Supernova remnants (SNRs) are known to accelerate cosmic-rays (CRs) to relativistic energies [1], where it is assumed that the highest energies are reached during the early times of the SNR evolution [2].

However, the link between the acceleration of CRs in SNRs and their release into the interstellar medium (ISM) has still to be build. Recent models for CR acceleration that include the CR-feedback on the plasma-flow as well as the amplification of magnetic turbulence are either steady-state calculations (e.g [3]) or include particle in cell codes that cover only very short timescales[4]. The late phases of the remnant evolution are poorly studied although they span a huge part of the SNRs lifetime. The possibility of reacceleration of CRs at slow shocks [5] has be discussed as well as the modification of the CR spectra due to an enhanced escape-rate of CRs[6].

In this work we present a time-dependent, test-particle calculation of the CR acceleration over the whole lifetime of a SNR including the radiative phase. We show that the maximum energy of the accelerated CRs differs only by one order of magnitude and that the late evolution stages give rise to the formation of some spectral features.

\section{Basic equations and assumptions}

\subsection{Cosmic rays}

We model the acceleration of cosmic rays using a kinetic approach in the test-particle approximation $[7,8,9]$, thus the CR-pressure has always to stay below $10 \%$ of the shock ram pressure. The time-dependent transport equation for the differential number density of cosmic rays $N$ [10] is given by:

$$
\frac{\partial N}{\partial t}=\nabla\left(D_{r} \nabla N-\mathbf{u} N\right)-\frac{\partial}{\partial p}\left((N \dot{p})-\frac{\nabla \cdot \mathbf{u}}{3} N p\right)+Q,
$$

where $D_{r}$ denotes the spatial diffusion coefficient, $\mathbf{u}$ the advective velocity, $\dot{p}$ energy losses and $Q$ the source of thermal particles.

We solve this transport equation in a frame co-moving with the shock. The radial coordinate is transformed according to $(x-1)=\left(x^{*}-1\right)^{3}$, where $x=r / R_{s h}$. For a equidistant binning of $x^{*}$ this transformation guarantees a very fine resolution close to the shock and a outer grid-boundary that extends to several tens of shock-radii upstream for $x^{*}>>1$. Thus all accelerated particles can be kept in the simulation domain.

Following [8] we apply Bohm diffusion close to the shock and have a transition to the galactic diffusion coefficient further upstream. The code contains the possibility consider the amplification of magnetic turbulence and thus calculate the diffusion coefficient self-consistent[11] but this will be addressed in future work. 


\subsubsection{Injection}

We inject particles according to the thermal leakage injection model[12]. Here the efficiency of injection $\eta_{i}$ is given by

$$
\eta_{i}=\frac{4}{3 \sqrt{\pi}}(\sigma-1) \psi^{3} e^{-\psi^{2}}
$$

where $\sigma$ is the shock compression ratio and $\psi$ is the multiple of the thermal momentum we inject particles at. Usually $\eta_{i}$ is assumed to be constant. The total number of injected particles might well change over time depending on the upstream density distribution. For a type1a SNR expanding in an uniform ISM the injection term in (2.1) will be growing with time due to the growing shocksurface. As a result the last injected particles are dominating the volume integrated particle spectra.

However, there have been several cases discussed in which $\eta_{i}$ might be or have been changing with time[13, 14]. To investigate the effects of a time-dependent injection efficiency, we included to possibility to vary $\eta_{i}$ according to $\eta_{i, t}(t)=\eta_{i}\left(\frac{t}{t_{0}}\right)^{a}$.

\subsection{Hydrodynamics}

The evolution of a SNR without CR-feedback can be described solving the standard gasdynamical equations

$$
\begin{array}{r}
\frac{\partial}{\partial t}\left(\begin{array}{c}
\rho \\
\mathbf{m} \\
E
\end{array}\right)+\nabla\left(\begin{array}{c}
\rho \mathbf{v} \\
\mathbf{m} \mathbf{v}+P \mathbf{I} \\
(E+p) \mathbf{v}
\end{array}\right)^{T}=\left(\begin{array}{l}
0 \\
0 \\
L
\end{array}\right) \\
\frac{\rho \mathbf{v}^{2}}{2}+\frac{P}{\gamma-1}=E
\end{array}
$$

where $\rho$ is the density of the thermal gas, $\mathbf{v}$ the plasma velocity, $\mathbf{m}=\mathbf{m} \rho$ the momentum density, $P$ the thermal pressure of the gas, $L$ the energy losses due to cooling and $E$ the total energy of the ideal gas with $\gamma=5 / 3$. This system of equations is solved under the assumption of a spherical symmetry in 1-D using the PLUTO-code [15]. The non-equilibrium cooling function $L$ is taken from [16].

In this work we consider type1a supernova explosions. Therefore we apply

$$
\begin{array}{r}
\rho_{S N}=A \exp \left(-v / v_{e}\right) t_{i}^{-3} \text { and } v=r / t_{i} \text { with } \\
v_{e}=\left(\frac{E_{e x}}{6 M_{e j}}\right)^{1 / 2} \text { and } A=\frac{6^{3 / 2}}{8 \pi} \frac{M_{e j}^{5 / 2}}{E_{e x}^{3 / 2}}
\end{array}
$$

as initial conditions[17]. Here $t_{i}$ is the time that has passed since the SN explosion and thus the starting time of our simulation, $M_{e j}$ the ejecta mass, $E_{e x}$ the explosion energy and $r$ the spatial coordinate.

For our simulations, we choose an initial age of about three months, as the solution converges rather fast against solutions with an lower initial age. The ejecta mass was 1.4 solar masses and the density of the ambient medium $0.4 \mathrm{~cm}^{-3}$. 


\section{Results}

Using the framework described above, we followed the evolution of a SNR for 100000 years. The remnant entered the Sedov phase after $\approx 1300$ years, the radiative phase after $\approx 35000$ years. After $\approx 85000$ years the shock compression ratio started to drop below 4 and reached a value of 3.6 when the simulation stopped. The shock speed dropped from $\approx 13000 \mathrm{~km} / \mathrm{s}$ to $\approx 45 \mathrm{~km} / \mathrm{s}$ at the end of the simulation.

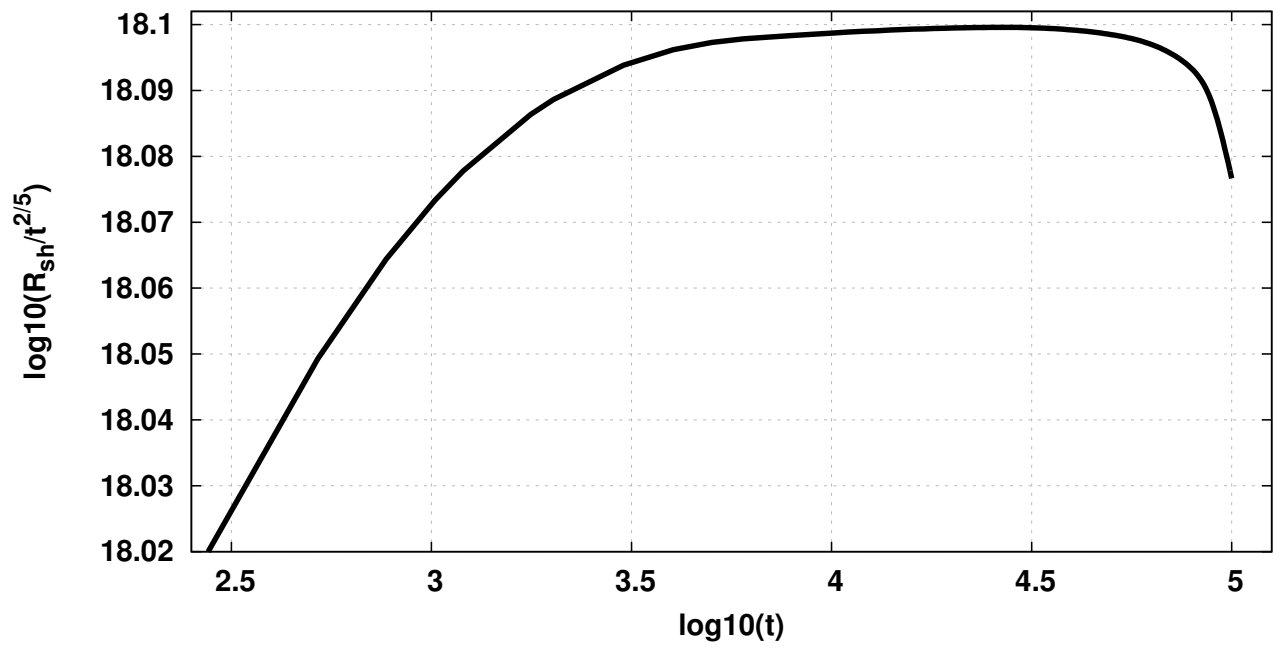

Figure 1: The curve shows the radial position of the SNRs forward shock over time scaled with $t^{2 / 5}$. The transitions between free-expansion and Sedov phase and Sedov and radiative phase appear at around 1300yrs and 35000yrs respectively.

In Fig. 1 one can see the different stages of evolution the remnant went through. The transition to the Sedov phase happens when the swept-up mass is $\approx 10 M_{\text {sol }}$.

It is important to point out, that after 10000 years the shock speed has already dropped to $\approx 600 \mathrm{~km} / \mathrm{s}$. Thus the speed difference between the end of the Sedov phase and the end of the radiative phase is just one order of magnitude. As a result the maximum energy, that can be reached during the radiative phase, should be just one order of magnitude lower than that during the Sedov phase.

\subsection{Static diffusion coefficient}

The evolution of the volume integrated downstream spectra is shown in Fig. 2. In the case of the steady injection the downstream spectra reassemble powerlaws with $s=-2$ up to 50000 years. The spectra at 100000 years feature some curvature. Responsible are two phenomena that operate successively. First the total compression ratio rises when the remnant enters the radiative phase. This higher compression ratio is felt by the highest energetic particles which thus show a harder spectrum. When the forward shock gets weaker, the lowest energetic particles experience a lower compression ratio, thus their spectrum becomes softer. This effect is assisted by an increasing density at the forward shock due to the formation of an dense shell which also results in a softer spectral index for the low energetic particles. 


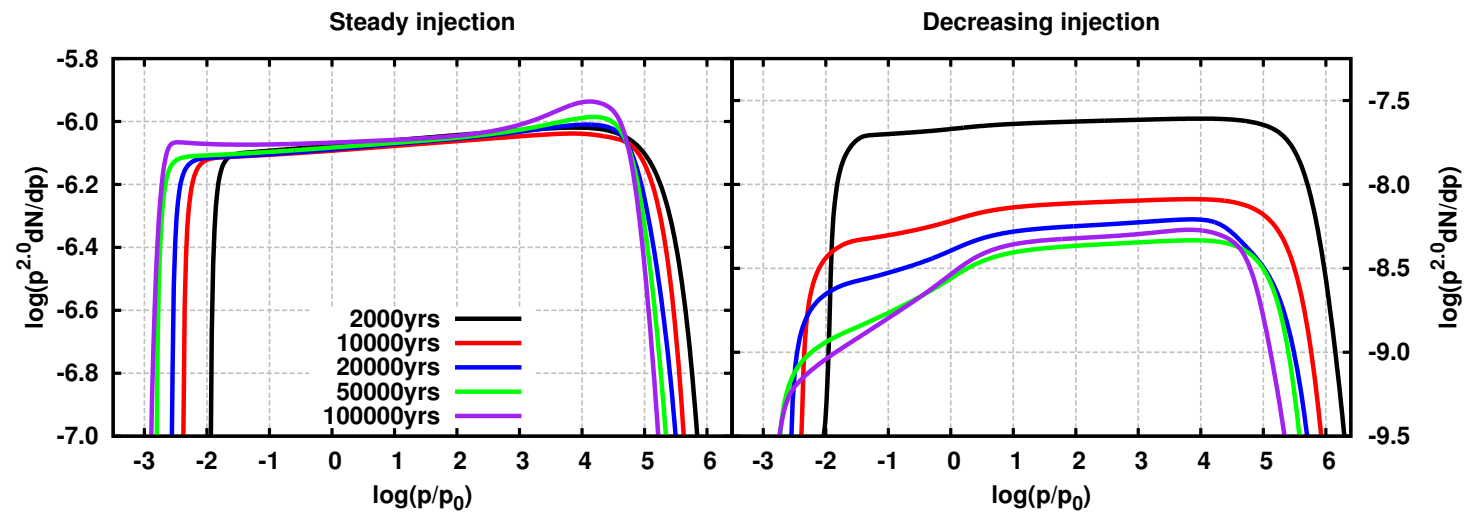

Figure 2: The left panels show the downstream proton spectrum in the case of steady injection, while the right shows the case of decreasing injection with $\eta_{i, t} \propto t^{-1}$.

The spectra for the case of an decreasing injection show a different spectral behaviour. Here from 10000 years on a spectral break at around $3 \mathrm{GeV}$ can be seen. Particles with lower energies show harder spectra whereas particles with higher energies show $s=-2$ spectra. Also here a small hardening at the highest energies can be seen due to the increased total compression ratio during the radiative phase. At the lowest energies the spectral hardening due to the decreasing injection is dominant. This is qualitatively in agreement with [13].

The upstream spectra almost reassemble log-parabolas, because of the small variation of the maximum energy of one order of magnitude. The spectrum for the case of decreasing injection is flattened an reassembles a power law in a small energy band due to the decreasing density of the CRs escaping.

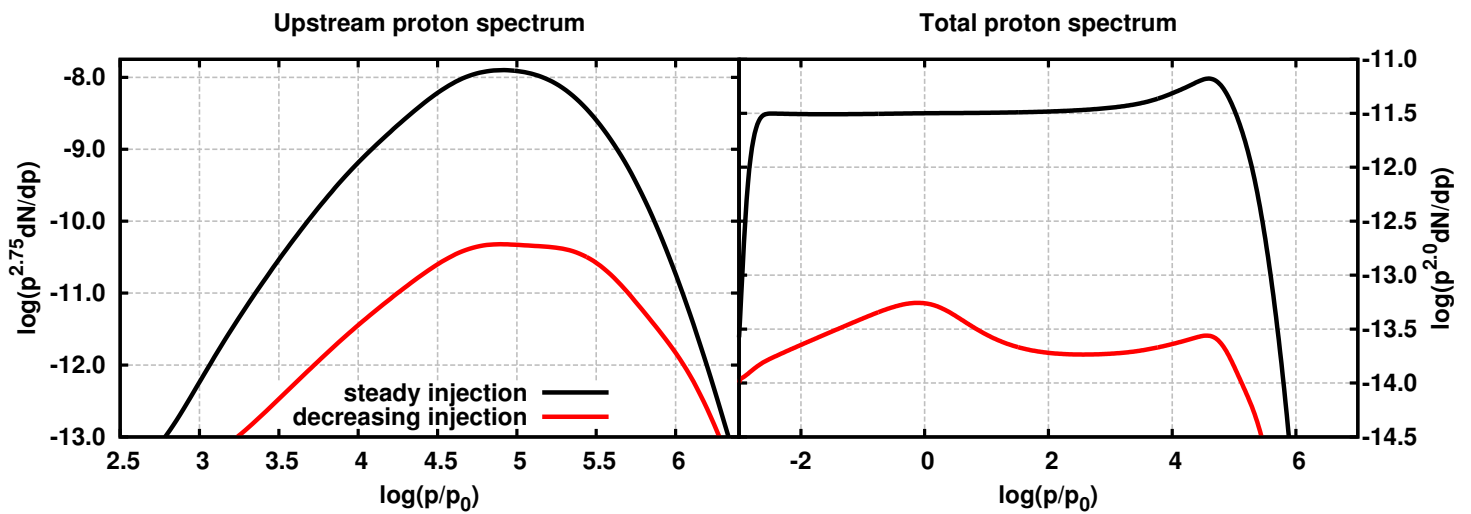

Figure 3: Proton spectrum for model with Bohm diffusion after 100kyrs. The left panel shows the volume integrated upstream proton spectrum and the right the volume integrated proton spectrum in the total simulation volume. The black lines correspond to a model with steady injection, while the red ones to one with a decreasing injection.

The total spectra, integrated over the whole simulation reassemble almost perfect $s=-2$ spectra for the case of static injection with a spectral hardening at the highest energies. The spectrum 
for the case of decreasing injection is more complicated. The high energy part is similar to the case of constant injection. The low energetic particles produced during the late phases of the remnant evolution, when the dense shell forms, show a harder spectrum and then a soft transition to the $s=-2$ spectrum at around $10 \mathrm{GeV}$. In general is the shape of the spectrum dominated by the decreasing injection, rather than the properties of the radiative shock.

\section{Conclusions}

We developed a model for the particle acceleration in SNRs up to a point where the forward shock of the remnant starts to become a weak shock by solving the time-dependant transport equation for the CRs in a test-particle limit. In this work we considered a static Bohm-like diffusion coefficient upstream of the forward shock, whereat it is planned to consider the amplification of turbulence as a next step and study the effect of the enhanced escape-rate.

The particle spectra for steady injection after 100000 years show deviations from standard DSA-spectra by a hardening of the spectrum at the highest energies due to the increased total compression ratio during the radiative phase and a softening at the lowest energies due to the weakening of the shock after the radiative phase. Furthermore is the difference in maximum particle energy just one order of magnitude between the Sedov phase and the end of the radiative phase. The spectra for a decreasing injection are more complicated and demonstrate qualitatively the effects a non steady injection might have on the observed and released particle spectra.

With our model the creation of a link between the acceleration of CRs and their release into the ISM is in reach.

\section{References}

[1] M. Ackermann, M. Ajello, Allafort, et al. Detection of the Characteristic Pion-Decay Signature in Supernova Remnants. Science, 339:807-811, February 2013.

[2] G. Morlino. Supernova Remnant-Cosmic Ray connection: a modern view. ArXiv e-prints, June 2017.

[3] A. M. Bykov, D. C. Ellison, S. M. Osipov, and A. E. Vladimirov. Magnetic Field Amplification in Nonlinear Diffusive Shock Acceleration Including Resonant and Non-resonant Cosmic-Ray Driven Instabilities. APJ, 789:137, July 2014.

[4] D. Caprioli, P. Blasi, E. Amato, and M. Vietri. Dynamical feedback of self-generated magnetic fields in cosmic ray modified shocks. MNRAS, 395:895-906, May 2009.

[5] M. Cardillo, E. Amato, and P. Blasi. Supernova remnant W44: a case of cosmic-ray reacceleration. aap, 595:A58, October 2016.

[6] M. A. Malkov, P. H. Diamond, and R. Z. Sagdeev. Nature Communications, 2:194, February 2011.

[7] I. Telezhinsky, V. V. Dwarkadas, and M. Pohl. Astroparticle Physics, 35:300-311, January 2012. 
[8] I. Telezhinsky, V. V. Dwarkadas, and M. Pohl. A\&A, 541:A153, May 2012.

[9] I. Telezhinsky, V. V. Dwarkadas, and M. Pohl. Acceleration of cosmic rays by young corecollapse supernova remnants. aap, 552:A102, April 2013.

[10] J. Skilling. MNRAS, 172:557-566, September 1975.

[11] R. Brose, I. Telezhinsky, and M. Pohl. Transport of magnetic turbulence in supernova remnants. aap, 593:A20, August 2016.

[12] S. Gabici, P. Blasi, and G. Vannoni. In T. Bulik, B. Rudak, and G. Madejski, editors, Astrophysical Sources of High Energy Particles and Radiation, volume 801 of American Institute of Physics Conference Series, pages 369-372, November 2005.

[13] O. Petruk, S. Orlando, M. Miceli, and F. Bocchino. Linking gamma-ray spectra of supernova remnants to the cosmic ray injection properties in the aftermath of supernovae. ArXiv e-prints, July 2017.

[14] H. J. Völk, E. G. Berezhko, and L. T. Ksenofontov. Variation of cosmic ray injection across supernova shocks. aap, 409:563-571, October 2003.

[15] A. Mignone, G. Bodo, S. Massaglia, T. Matsakos, O. Tesileanu, C. Zanni, and A. Ferrari. PLUTO: A Numerical Code for Computational Astrophysics. apjs, 170:228-242, May 2007.

[16] R. S. Sutherland and M. A. Dopita. Cooling functions for low-density astrophysical plasmas. apjs, 88:253-327, September 1993.

[17] V. V. Dwarkadas and R. A. Chevalier. Interaction of Type IA Supernovae with Their Surroundings. apj, 497:807-823, April 1998. 